\title{
Uptake of water from soils by plant roots
}

\author{
P. A. C. Raats
}

Published online: 17 January 2007

(C) Springer Science+Business Media B.V. 2006

\begin{abstract}
Uptake of water by plant roots can be considered at two different Darcian scales, referred to as the mesoscopic and macroscopic scales. At the mesoscopic scale, uptake of water is represented by a flux at the soil-root interface, while at the macroscopic scale it is represented by a sink term in the volumetric mass balance. At the mesoscopic scale, uptake of water by individual plant roots can be described by a diffusion equation, describing the flow of water from soil to plant root, and appropriate initial and boundary conditions. The model involves at least two characteristic lengths describing the root-soil geometry and two characteristic times, one describing the capillary flow of water from soil to plant roots and another the ratio of supply of water in the soil and uptake by plant roots. Generally, at a certain critical time, uptake will switch from demand-driven to supply-dependent. In this paper, the solutions of some of the resulting mesoscopic linear and nonlinear problems are reviewed. The resulting expressions for the evolution of the average water content can be used as a basis for upscaling from the mesoscopic to the macroscopic scale. It will be seen that demand-driven and supply-dependent uptake also emerge at the macroscopic scale. Information about root systems needed to operationalize macroscopic models will be reviewed briefly.
\end{abstract}

Keywords Falling rate - Richards equation - Root length density ·

Uptake of water $\cdot$ Steady state approximation $\cdot$ Steady rate approximation

\section{Introduction}

1.1 Theory of Richards for flow of water in unsaturated soils

About 70 years ago, Lorenzo A. Richards consolidated the efforts of previous generations of soil physicists by formulating a general, macroscopic theory for movement

P. A. C. Raats $(\bowtie)$

Wageningen University and Research Centre, Paaskamp 16, 9301 KL Roden, The Netherlands e-mail: pac.raats@home.nl 
of water in rigid, unsaturated soils (Richards 1931). Richards theory fits experience in many branches of continuum mechanics. It combines the balance of mass, expressed in the equation of continuity, and of momentum, expressed in Darcy's law:

$$
\begin{aligned}
& \frac{\partial \bar{\theta}}{\partial t}=-\nabla \cdot(\bar{\theta} \mathbf{v})-\bar{\lambda}, \\
& \bar{\theta} \mathbf{v}=-\bar{k} \nabla \bar{h}+\bar{k} \nabla z,
\end{aligned}
$$

where $t$ is the time, $\nabla$ is the vector differential operator, $\bar{\theta}$ is the volumetric water content, $\overline{\mathbf{v}}$ is the velocity of the water, $\bar{\theta} \mathbf{v}$ is the volumetric flux, $\bar{\lambda}$ is the volumetric rate of uptake of water by plant roots (see Sect. 1.2), $\bar{k}$ is the hydraulic conductivity, $\bar{h}$ is the capillary pressure head, and $z$ is the vertical coordinate taken positive downward. The bars above the symbols denote that at this stage the scale of discourse is based on macroscopic volume elements containing representative amounts of roots, such that the uptake can be represented by a volumetric sink term $\bar{\lambda}$ in the volumetric water balance (1).

The capillary pressure head $\bar{h}$ is defined by

$$
\bar{h}=\frac{\left(\bar{p}_{w}-\bar{p}_{g}\right)}{\gamma_{w} g}=-\frac{\bar{p}_{c}}{\gamma_{w} g},
$$

where $\bar{p}_{w}$ and $\bar{p}_{g}$ are the pressures of the aqueous and gaseous phases, $\bar{p}_{c}$ is the capillary pressure, $\gamma_{w}$ is the mass density of the water, and $g$ is the magnitude of the gravitational force per unit mass. In the theory of Richards, it is assumed that the pressure of the gaseous phase is spatially uniform and constant. The capillary pressure head $\bar{h}$ and the hydraulic conductivity $\bar{k}$ are highly nonlinear functions of the volumetric water content $\bar{\theta}$. A further complicating factor is that the relationship $\bar{h}(\bar{\theta})$ is hysteretic.

For processes not involving hysteresis, Darcy's law can also be written as

$$
\bar{\theta} \mathbf{v}=-\bar{D} \nabla \bar{\theta}+\bar{k} \nabla z
$$

or

$$
\bar{\theta} \mathbf{v}=-\nabla \bar{\varphi}+\bar{k} \nabla z
$$

where $\bar{D}=\bar{k} \mathrm{~d} \bar{h} / \mathrm{d} \bar{\theta}$ is the soil water diffusivity, and $\bar{\varphi}$ is the matric flux potential defined by

$$
\bar{\varphi}-\bar{\varphi}_{\mathrm{ref}}=\int_{\bar{h}_{\mathrm{ref}}}^{\bar{h}} \bar{k} \mathrm{~d} \bar{h}=\int_{\bar{\theta}_{\mathrm{ref}}}^{\bar{\theta}} \bar{D} \mathrm{~d} \bar{\theta},
$$

where the subscript ref denotes a reference state. The volumetric flux $\bar{\theta} \mathbf{v}$ is the sum of a matric component $-\bar{k} \nabla \bar{h}=-\bar{D} \nabla \bar{\theta}=-\nabla \bar{\varphi}$ and a gravitational component $\bar{k} \nabla z$. The matric component of the volumetric flux is given by the gradient of $\bar{\varphi}$ and therefore it is appropriate to call $\bar{\varphi}$ the matric flux potential (Raats 1970). A transformation of 
the type (6) was given around 1880 by Kirchhoff in his lectures on heat conduction (Kirchhoff 1894). For this reason, often $\bar{\varphi}$ is called the Kirchhoff potential and the transformation from $\bar{h}$ or $\bar{\theta}$ to $\bar{\varphi}$ is referred to as the Kirchhoff transformation.

The theory of Richards can be justified on the basis of the principles of surface tension and viscous flow at the pore scale (Miller and Miller 1956, Whitaker 1986). It can also be formulated within the framework of the modern continuum theory of mixtures (Truesdell and Toupin 1960), provided that one recognizes from the outset the existence of the separate solid, liquid, and gaseous phases (Raats 1984, 1998).

In the context of Richards equation, the relationships among the water content $\bar{\theta}$, pressure head $\bar{h}$, and hydraulic conductivity $\bar{k}$ define the hydraulic properties of a soil. Two major conferences on physical characterization were organized in 1989 and 1997 by the U.S. Salinity Laboratory and the University of California at Riverside (Raats 1992, Van Genuchten et al. 1999). One can distinguish two groups of parametric expressions describing hydraulic properties (Raats 2001, Raats et al. 2002).

The first group yields flow equations that can be solved analytically, either directly or following one or more transformations, in most cases as a result of linearization following one or more transformations. To the first group belong:

- the class of linear soils with the diffusivity $\bar{D}=\bar{k} \mathrm{~d} \bar{h} / \mathrm{d} \bar{\theta}$ constant and the hydraulic conductivity $\bar{k}$ linear in $\bar{\theta}$;

This leads to a linear Fokker-Planck equation, which can be solved relatively easily. It yields useful results for integral aspects of the water balance, but is rather unreliable with respect to details of the distribution of the water content. As will be seen in this paper, the class of linear soils has regularly been considered in problems involving uptake of water

- the class of Brooks-Corey power function soils.

For this class of soils $\bar{h}, \bar{k}$, and $\bar{D}$ are all power functions of $\bar{\theta}$. In Sect. 4.4.2 of this paper the power function $\bar{D}(\bar{\theta})$ relationship is used:

$$
\bar{D}=\left(\bar{D}_{\mathrm{s}} / \bar{\theta}_{\mathrm{s}}^{n}\right) \bar{\theta}^{n},
$$

where $\bar{\theta}_{\mathrm{S}}$ is the water content at saturation and $\bar{D}_{\mathrm{s}}$ the corresponding diffusivity. The class of Brooks-Corey power function soils has turned up regularly in recent years as resulting from fractal models of pore structure. It also plays a key role in the mathematical literature on the so-called porous medium equation, i.e. the nonlinear diffusion equation with the diffusivity a power function of the volumetric water content, and on the corresponding special case of the Richards equation (Gilding 1991). The main interest has been in so-called similarity solutions.

Other classes of soils, belonging to the first group, and associated flow problems are discussed in recent review papers (Raats 2001, Raats et al. 2002).

The second group of parametric expressions describing the soil hydraulic properties is favored in numerical studies and to a large extent shares flexibility with a rather sound basis in Poiseuillean flow in networks of capillaries. In this group of relationships among the water content $\bar{\theta}$, pressure head $\bar{h}$, and hydraulic conductivity $\bar{k}$, the latter is calculated from the water retention characteristic and certain assumptions concerning the geometry of the pore system (Raats 1990b, 1992). The procedure in essence links physico-mathematical models at the Darcy and Navier-Stokes scales. By introducing a very general equation for the water retention characteristic and a rather general predictive model for the relative hydraulic conductivity, various well known 
classes of soils can be regarded as subclasses of one and the same superclass, thus summarizing a widely scattered literature. The subclasses include those of Brooks-Corey and of Van Genuchten-Mualem as special cases.

\subsection{Uptake of water by plant roots at different scales}

The volumetric rate of uptake $\bar{\lambda}$ in Eq. 1 represents the uptake averaged over a large number of roots. The uptake of water by the plant roots is induced by the flow inside the plant to the above-ground evaporating surfaces. Where the water is taken up depends on the spatial distribution of the roots and of the water status of the soil, and perhaps also on the resistance to water flow in the plant. If the other terms in (1) are determined, then $\bar{\lambda}$ can be calculated. The term $\partial \bar{\theta} / \partial t$ can be evaluated from measurements of the water content in the course of time. The term $\nabla \cdot(\overline{\theta \mathbf{v}})$ can be calculated on the basis of Darcy's law, making use of measurements of the $\bar{k}(\bar{\theta})$ relationship of the soil and measurements of the pressure head $\bar{h}$ in the course of time. A detailed review of this procedure to determine the spatial distribution of $\bar{\lambda}$ is presented in Raats (1982).

If the crop or vegetation has a certain demand for water and the water status of the soil is such that the demand can be met at all times, then it may be appropriate to simply assume that the spatial and temporal distribution of the uptake is given by

$$
\bar{\lambda}(\mathbf{x}, t)=f(\mathbf{x}, t) T(t)
$$

where $T$ is the rate of transpiration and $f(\mathbf{x})$ is the distribution function for uptake of water by plant roots. In this connection the distribution function

$$
f(x, y, z)=\delta^{-1} \exp (-z / \delta),
$$

where $\delta$ can be interpreted as an equivalent rooting depth, has turned out to be rather useful (Raats 1974a).

The uptake process becomes more complex when the soil is too dry for the flow to the plant roots to satisfy the evaporative demand. In water balance models limited availability of water is often accounted for by empirical equations. These equations are partly based on experience and partly on models of flow of water to individual roots. The main purpose of the remainder of this paper is to review and extend the latter models. Individual roots function at a mesoscopic scale, which is intermediate between the microscopic Navier-Stokes scale and the macroscopic scale considered in Eqs. 1 and 2. It is common practice to use the same physical model of capillary flow at the macroscopic and mesoscopic scales. The differences are:

- At the macroscopic scale, the uptake by plant roots is represented by a sink term $\bar{\lambda}$ in the volumetric mass balance, while at the mesoscopic scale it is represented by a flux at the soil-root interface.

- The variables and parameters at the macroscopic scale introduced in Sect. 1 were denoted by bars. For the corresponding parameters and variables at the mesoscopic scale these bars are omitted.

- In Darcy's law, the gravitational force is likely to be important at the macroscopic scale, but unlikely to be important at the mesoscopic scale.

The remainder of this paper mainly concerns the derivation of expressions for the rate of uptake $\bar{\lambda}$ at the macroscopic scale from an analysis of flow at the mesoscopic scale. 


\section{Description of root systems}

In the literature, a variety of parameters have been proposed to describe aspects of the geometry of plant roots. In the following, first some commonly used geometric parameters and relationships among them are presented. Next the geometrical parameters commonly used in soil-plant-atmosphere models are related to the root mass based parameters often measured by ecologists.

\subsection{Geometry of root systems}

For an individual plant root, two characteristic lengths can be identified:

$-r_{0}$, radius of the plant root and also the internal radius of the equivalent cylindrical shell of soil associated with the plant root;

- $r_{1}$, external radius of the equivalent cylindrical shell of soil associated with the plant root.

For a system of plant roots distributed in soil, the three following parameters can be used:

- $L$, the root length per unit volume;

- $\sigma$, the root surface area per unit volume;

- $\phi_{\mathrm{r}}$ and $\phi_{\mathrm{s}}$, the root and soil volume fractions, subject to the constraint

$$
\phi_{\mathrm{r}}+\phi_{\mathrm{s}}=1 .
$$

For spatially uniformly distributed roots, the root length density $L$ is equal for the inverse of the volume of roots plus soil associated with unit length of root:

$$
L=\left(\pi r_{1}^{2}\right)^{-1} \text {. }
$$

The root volume fraction $\phi_{\mathrm{r}}$ is equal to the ratio of the volume of a unit length of root and the volume of this unit length of root plus the cylindrical shell of soil associated with it:

$$
\phi_{\mathrm{r}}=\left(r_{0} / r_{1}\right)^{2}=\pi r_{0}^{2} L .
$$

The constraint (10) on the volume fractions implies:

$$
\phi_{\mathrm{s}}=1-\phi_{\mathrm{r}}=1-\left(r_{0} / r_{1}\right)^{2}=1-\pi r_{0}^{2} L .
$$

The root surface area $\sigma$ is equal to the ratio of the surface area of a unit length of root divided by the volume of root plus the cylindrical shell of soil associated with unit length of root:

$$
\sigma=2 r_{0} / r_{1}^{2}=2 \pi r_{0} L=2\left(\pi \phi_{\mathrm{r}} L\right)^{1 / 2} .
$$

From (11) and (14) it follows that

$$
r_{0}=\sigma(2 \pi L)^{-1},
$$

and

$$
r_{1}=(\pi L)^{-1 / 2}=\left(\frac{2 r_{0}}{\sigma}\right)^{1 / 2} .
$$


Clearly, the characterizations of a uniform root system by the radii $r_{0}$ and $r_{1}$ and by the root length density $L$ and the specific root surface area $\sigma$ are equivalent. Solving Eq. 12 for $r_{0} / r_{1}$ gives:

$$
r_{0} / r_{1}=\phi_{\mathrm{r}}^{1 / 2}
$$

This shows that, to characterize the geometry of a uniform root system, either the radii $r_{0}$ and $r_{1}$, or two of the parameters $L, \sigma$, and $\phi_{\mathrm{r}}$ or $\phi_{\mathrm{s}}$ can be used. In experimental practice, the root length density $L$ and the root radius $r_{0}$ are usually measured. Then Eq. 16 can be used to calculate $r_{1}$ and Eqs. 12-14 can in turn be used to calculate $\sigma$, $\phi_{\mathrm{r}}$ and $\phi_{\mathrm{s}}$.

\subsection{Spatial-temporal description and interconversion of bulk root mass density} and geometrical parameters

Root water uptake is strongly related to the geometrical parameters root length density and root radius. Such root system data are scarce and therefore it is of interest to relate more widely available root mass density data to these geometrical parameters. This will also clarify the different ways researchers describe root systems.

Multiplication of the root volume fraction $\phi_{\mathrm{r}}$ by the intrinsic mass density $\gamma_{\mathrm{r}}$, i.e. the mass per unit volume of root tissue, gives the macroscopic root mass density $\mu$. Together with (12) this definition gives:

$$
\mu=\gamma_{\mathrm{r}} \phi_{\mathrm{r}}=\gamma_{\mathrm{r}}\left(r_{0} / r_{1}\right)^{2}=\gamma_{\mathrm{r}} \pi r_{0}^{2} L .
$$

The factor $\pi r_{0}^{2} \gamma_{\mathrm{r}}$ in (18) represents the root mass per unit length of root. Van Noordwijk (1987) refers to it as the specific root length. A complicating factor related to the intrinsic density $\gamma_{\mathrm{r}}$ is the often considerable gaseous volume fraction of root tissue, in particular in plants adapted to growing in wet soils (Van Noordwijk and Brouwer 1991). Also, in view of the variance of the root radii, Van Noordwijk and Brouwer (1991) propose the use in (18) of the square of the quadratic average root radius which is equal to the square of the linear average root radius plus the variance of the root radius. Equation 18 relates the geometrical parameters root radius $r_{0}$ and root length per unit volume $L$ used in water uptake models to the bulk root mass volumetric density $\mu$ often measured by ecologists. Equation 18 implies that it is important that root system databases for use in specific types of soil-water-plant-atmosphere models should not be restricted to the spatial-temporal distribution of $\mu(z, t)$. They should in addition also contain some information on the intrinsic density $\gamma_{\mathrm{r}}$ and the root radii $r_{0}$.

In the following two sub-subsections various models for the spatial-temporal distribution of roots are reviewed.

\subsubsection{The power law model of Gale and Grigal}

Gale and Grigal (1987) introduced the following expression for a time-invariant cumulative root biomass fraction as a function of depth:

$$
Y(z)=\frac{B(z, t)}{B_{\infty}(t)}=1-\beta^{-z / \delta},
$$

where $B(z, t)$ is the root biomass per unit area of land above depth $z$ at time $t$, $B_{\infty}(t)=B(\infty, t)$ is the total root biomass per unit area of land at time $t$, and $\beta$ and $\delta$ Springer 
are vegetation dependent parameters. Equation 19 has been widely used to represent depth-cumulative root biomass distribution data (Jackson 1999; Jackson et al. 1996, 1997; Feddes et al. 2001; Zeng 2001; Zeng et al. 1998). To render those data useful in root water uptake models, they should be converted first to spatial-temporal distributions of the bulk root mass density $\mu(z, t)$ and next to spatial-temporal distributions of the root length density $L(z, t)$.

Partial differentiation of $B(z, t)$ with respect to $z$ gives an expression for the bulk root mass density $\mu(z, t)$ :

$$
\mu(z, t)=\frac{\partial B(z, t)}{\partial t}=\frac{\mathrm{d} Y(z)}{\mathrm{d} z} B_{\infty}(z)=\delta^{-1}(\ln \beta) B_{\infty}(t) \beta^{-z / \delta} .
$$

Equation 20 implies that the surface root biomass density $\mu(0, t)=\delta^{-1}$ $(\ln \beta) B_{\infty}(t)$. Note that an equivalent uniform root system with root biomass density $\mu(z, t)=\mu(0, t)=\delta^{-1}(\ln \beta) B_{\infty}(t)$ in the depth range $0 \leqslant z \leqslant \delta(\ln \beta)^{-1}$ will have the same total root biomass $B_{\infty}(t)$. Thus $\delta(\ln \beta)^{-1}$ in (20) can be interpreted as the characteristic rooting depth based on the surface root biomass density and the total root biomass.

Equation 20 implies that, at any time $t$ and at any position $z$, the relative time rate of change of the root biomass density $\mu(z, t)$ is equal to the relative time rate of change of the total root biomass $B_{\infty}(t)$ :

$$
\frac{1}{\mu(z, t)} \frac{\partial \mu(z, t)}{\partial t}=\frac{1}{B_{\infty}(t)} \frac{\mathrm{d} B_{\infty}(t)}{\mathrm{d} t} .
$$

Equation 21 shows that the model of Gale and Grigal (1987) implies an allometric relationship between $\mu(z, t)$ and $B_{\infty}(t)$, independent of time $t$ and depth $z$. The model also implies that roots are present at all depths $z>0$ at all times $t>0$. Especially for crops and vegetations in the early stages this will not be realistic.

Solving (18) for the root length density $L$ and introducing (20) for the root mass density $\mu(z, t)$ gives:

$$
L(z, t)=\frac{\mu(z, t)}{\pi r_{0}^{2} \gamma_{\mathrm{r}}}=\frac{\delta^{-1}(\ln \beta) B_{\infty}(t) \beta^{-z / \delta}}{\pi r_{0}^{2} \gamma_{\mathrm{r}}} .
$$

\subsubsection{The exponential model of Arora and Boer}

Arora and Boer (2003) developed a more versatile root distribution model by introducing two modifications in the model of Gale and Grigal (1987):

- They specialized the model by setting:

$$
\beta=e \quad \text { implying } \quad \ln \beta=\ln e=1 .
$$

- To allow for the common experience that rooting depth increases in the course of time, they replaced the constant parameter $\delta$ by a parameter $\delta_{t}$ with a power-law dependence upon the total root biomass $B_{\infty}(t)$ :

$$
\delta_{t}=\delta B_{\infty}^{\alpha}(t),
$$

where $\delta$ and $\alpha$ are constants. 
Introducing (23) and (24) in, respectively, (20) and (19) gives:

$$
\begin{gathered}
\mu(z, t)=\delta_{t}^{-1} B_{\infty}(t) \exp \left(-z / \delta_{t}\right)=\delta^{-1} B_{\infty}^{1-\alpha}(t) \exp \left(-z /\left(\delta B_{\infty}^{\alpha}(t)\right)\right), \\
Y(z, t)=\frac{B(z, t)}{B_{\infty}(t)}=1-\exp \left(-z / \delta_{t}\right)=1-\exp \left(-z /\left(\delta B_{\infty}^{\alpha}(t)\right)\right) .
\end{gathered}
$$

Equation 25 implies that the surface root biomass density $\mu(0, t)=$ $\delta_{t}^{-1} B_{\infty}(t)=\delta^{-1} B_{\infty}^{1-\alpha}(t)$. An equivalent uniform root system with root biomass density $\mu(z, t)=\delta_{t}^{-1} B_{\infty}(t)=\delta^{-1} B_{\infty}^{1-\alpha}(t)$ in the depth range $0 \leqslant z \leqslant \delta_{t}=\delta B_{\infty}^{\alpha}(t)$ will have the same total root biomass $B_{\infty}(t)$. Thus the parameter $\delta_{t}=\delta B_{\infty}^{\alpha}(t)$ in (25) can be interpreted as the time dependent characteristic rooting depth based on the total root biomass $B_{\infty}(t)$ and the surface root biomass density $\delta^{-1} B_{\infty}^{1-\alpha}(t)$. Note that, unlike in (19), the cumulative root biomass fraction in (26) depends on time, whenever the total root biomass depends on time.

The relative time rate of change of the root biomass density corresponding to (25) is given by

$$
\begin{aligned}
\frac{1}{\mu(z, t)} \frac{\partial \mu(z, t)}{\partial t} & =\left[(1-\alpha)+\alpha z / \delta_{t}\right] \frac{1}{B_{\infty}(t)} \frac{\mathrm{d} B_{\infty}(t)}{\mathrm{d} t} \\
& =\left[(1-\alpha)+\alpha z /\left(\delta B_{\infty}^{\alpha}(t)\right)\right] \frac{1}{B_{\infty}(t)} \frac{\mathrm{d} B_{\infty}(t)}{\mathrm{d} t} \\
& =g(z, t) \frac{1}{B_{\infty}(t)} \frac{\mathrm{d} B_{\infty}(t)}{\mathrm{d} t} .
\end{aligned}
$$

Clearly, unlike in (21), in (27) the relative time rates of change of $\mu(z, t)$ and $B_{\infty}(t)$ are no longer equal to each other. Equation 27 shows that the model of Arora and Boer (2003) implies a spatially non-uniform and temporally variable allometric relationship between $\mu(z, t)$ and $B_{\infty}(t)$, depending on $\alpha, \delta, B_{\infty}(t)$ and $z$. Following Huxley (1972), we may refer to $g(z, t)$ in (27) as the growth coefficient. For a given time $t$ and depth $z, g(z, t)$ describes the relative time rate of change of the mass density $\mu(z, t)$ in comparison with the relative time rate of change of the total root biomass $B_{\infty}(t)$. However, note that the model of Arora and Boer (2003) still implies that roots are present at all depths $z>0$ and at all times $t>0$. Again, for crops and vegetations in the early stages this will not be realistic.

Solving (18) for the root length density $L(z, t)$ and introducing (25) for root mass density $\mu(z, t)$ gives:

$$
L(z, t)=\frac{\mu(z, t)}{\pi r_{o}^{2} \gamma_{\mathrm{r}}}=\frac{\delta^{-1} B_{\infty}^{1-\alpha}(t) \exp \left(-z /\left(\delta B_{\infty}^{\alpha}(t)\right)\right)}{\pi r_{0}^{2} \gamma_{\mathrm{r}}} .
$$

It is worthwhile to consider two special cases of the model of Arora and Boer (2003) with, respectively, $\alpha=0$ and $\alpha=1$.

If $\alpha=0,(24)$ implies that $\delta_{t}=\delta$ so that (25-28) reduce to

$$
\begin{gathered}
\mu(z, t)=\delta^{-1} B_{\infty}(t) \exp (-z / \delta), \\
Y(z, t)=1-\exp (-z / \delta), \\
\frac{1}{\mu(z, t)} \frac{\partial \mu(z, t)}{\partial t}=\frac{1}{B_{\infty}(t)} \frac{\mathrm{d} B_{\infty}(t)}{\mathrm{d} t},
\end{gathered}
$$




$$
L(z, t)=\frac{\mu(z, t)}{\pi r_{0}^{2} \gamma_{\mathrm{r}}}=\frac{\delta^{-1} B_{\infty}(t) \exp (-z / \delta)}{\pi r_{0}^{2} \gamma_{\mathrm{r}}} .
$$

Note that (29), (30) and (32) correspond to, respectively, (19), (20) and (22) with $\beta=e$ and that, not surprisingly, (31) is identical to (21). According to (31), for this special case of the model of Arora and Boer (2003) the allometric relationship between $\mu(z, t)$ and $B_{\infty}(t)$ is spatially uniform and temporally constant. Exponential dependence on depth has been considered earlier for the root distribution (Gerwitz and Page 1974) as well as the volumetric rate of water uptake (Raats 1974a; see Eqs. 8,9 above).

If $\alpha=1$, Eq. 24 implies that the characteristic rooting depth $\delta_{t}$ is proportional to the total root mass density $B_{\infty}(t)$ and (25-28) reduce to

$$
\begin{aligned}
& \mu(z, t)=\delta^{-1} \exp \left(-z /\left(\delta B_{\infty}(t)\right)\right), \\
& Y(z, t)=1-\exp \left(-z /\left(\delta B_{\infty}(t)\right)\right), \\
& \frac{1}{\mu(z, t)} \frac{\partial \mu(z, t)}{\partial t}=z /\left(\delta_{t}\right) \frac{1}{B_{\infty}(t)} \frac{\mathrm{d} B_{\infty}(t)}{\mathrm{d} t} \\
& =z /\left(\delta B_{\infty}(t)\right) \frac{1}{B_{\infty}(t)} \frac{\mathrm{d} B_{\infty}(t)}{\mathrm{d} t} \\
& =g(z, t) \frac{1}{B_{\infty}(t)} \frac{\mathrm{d} B_{\infty}(t)}{\mathrm{d} t}, \\
& L(z, t)=\frac{\mu(z, t)}{\pi r_{0}^{2} \gamma_{\mathrm{r}}}=\frac{\delta^{-1} \exp \left(-z /\left(\delta B_{\infty}(t)\right)\right)}{\pi r_{0}^{2} \gamma_{\mathrm{r}}} .
\end{aligned}
$$

Equation 33 implies that the surface root biomass density $\mu(0, t)=\delta$ is constant. An equivalent uniform root system with root biomass density $\mu(z, t)=\delta$ in the depth range $0 \leqslant z \leqslant \delta_{t}=\delta B_{\infty}(t)$ will have the same total root biomass $B_{\infty}(t)$. Thus $\delta B_{\infty}(t)$ in (33) can be interpreted as the time dependent characteristic rooting depth based on the surface root biomass density and the total root biomass. In other words, for $\alpha=1$ the surface root density is constant and the characteristic rooting depth increases linearly with the total root biomass $B_{\infty}(t)$. Note that in (35) the growth coefficient $g(z, t)$ is proportional to depth $z$ and inversely proportional to the total root biomass $B_{\infty}(t)$.

\section{Uptake of water by a root from a cylindrical shell of soil}

\subsection{Formulation in terms of dimensional variables}

The mesoscopic flow of water in the soil towards an individual plant root is described by the nonlinear diffusion equation resulting from a volumetric mass balance (1) and the diffusion form of Darcy's law (4):

$$
\frac{\partial \theta}{\partial t}=\frac{1}{r} \frac{\partial}{\partial r}\left[r D(\theta) \frac{\partial \theta}{\partial r}\right],
$$

where $r$ is the radial coordinate, $\theta$ is the volumetric water content at the mesoscopic scale in the region of influence of individual plant roots, and $D(\theta)$ is the soil water 
diffusivity. Note that, since the scale of discourse is now based on mesoscopic volume elements, no bars appear above $\theta$ and $D$. The initial distribution of the water content is assumed to be uniform:

$$
t=0, \quad r_{0}<r<r_{1}, \quad \theta=\theta_{i},
$$

where $\theta_{i}$ is the initial water content. The boundary condition at the outer boundary of the cylindrical shell of soil is:

$$
t>0, \quad r=r_{1}, \quad \frac{\partial \theta}{\partial r}=0 .
$$

The boundary condition at the soil-root interface is governed by the demand of water by the plant root and by the ability of the flow from the soil to the plant root to satisfy this demand. At the scale of the vegetation, consider a representative rooted volume $V$ of soil. The uptake $U$ of water from this volume of soil can be written as:

$$
U=\int_{V} \bar{\lambda} \mathrm{d} v=\int_{V}-F \sigma \mathrm{d} v=\int_{V} D(\theta) \frac{\partial \theta}{\partial r} \sigma \mathrm{d} v,
$$

where $F$ is the flux at the soil-root interface. If the flow of the water to the root is not limiting, the uptake $U$ is determined by the atmospheric conditions and the growth stage of the vegetation. If the root system is uniform, then at each point of the soil-root interface the flux can be written as:

$$
F=-\frac{U}{\sigma V}=-\frac{\bar{\lambda}}{\sigma}=-\frac{r_{1}^{2}}{2 r_{0}} \bar{\lambda}=-D(\theta) \frac{\partial \theta}{\partial r} .
$$

Clearly, increases of the specific root surface area $\sigma$ and the rooted volume $V$ reduce the demand $F$ on an individual root basis. For the boundary condition at the soil-root interface, two possibilities are considered:

A. during the period, up to the critical time $t_{\text {crit }}$, of constant rate of uptake $\bar{\lambda}_{c}$ :

$$
0<t<t_{\text {crit }}, \quad r=r_{o}, \quad D(\theta) \frac{\partial \theta}{\partial r}=\frac{\bar{\lambda}_{c}}{\sigma}=\frac{r_{1}^{2}}{2 r_{0}} \bar{\lambda}_{c} ;
$$

B. during the period, after the critical time $t_{\text {crit }}$, of falling rate of uptake $\bar{\lambda}$ :

$$
t>t_{\text {crit }}, \quad r=r_{o}, \quad \theta=\theta_{\text {crit }} .
$$

During the constant rate period, the overall mass balance at time $t$ requires that the total uptake of water per unit volume, $\bar{\lambda}_{c} t$, is equal to the average depletion per unit volume $\phi_{\mathrm{s}}\left(\theta_{i}-\bar{\theta}\right)$, so that

$$
\phi_{\mathrm{s}}\left(\theta_{i}-\bar{\theta}\right)=\bar{\lambda}_{c} t \quad \text { or } \quad t=\frac{\phi_{\mathrm{s}}\left(\theta_{i}-\bar{\theta}\right)}{\bar{\lambda}_{c}} .
$$

Equation 44 shows simply that during the constant rate period the average water content decreases linearly with time.

\subsection{Formulation in terms of dimensionless variables}

The problem formulated in the previous section will next be reformulated in terms of dimensionless variables. The dimensionless position $\rho$, time $\tau$, water depletion of the soil $\Theta$, and diffusivity $\Delta$ are, respectively, defined as: 


$$
\rho=\frac{r}{r_{1}}, \quad \tau=\frac{t}{t_{d}}, \quad \Theta=\frac{\theta_{i}-\theta}{\theta_{i}-\theta_{\text {crit }}}, \quad \Delta=\frac{D}{\widehat{D}},
$$

where the mean diffusivity $\widehat{D}$ and the characteristic time $t_{d}$ associated with the capillary flow of water are, respectively, defined as:

$$
\widehat{D}=\frac{\int_{\theta_{\text {crit }}}^{\theta_{i}} D \mathrm{~d} \theta}{\theta_{i}-\theta_{\text {crit }}}, \quad t_{d}=\frac{r_{1}^{2}}{\widehat{D}} .
$$

The characteristic time $t_{d}$ describes the capillary flow of water from the soil to the plant roots. A second characteristic time $t_{c}$ is defined as the ratio of the supply of water in the soil and the rate of uptake $\bar{\lambda}_{c}$ by plant roots during the constant rate period:

$$
t_{c}=\frac{\left(\theta_{i}-\theta_{\text {crit }}\right) \phi_{\mathrm{s}}}{\bar{\lambda}_{c}} .
$$

The characteristic time $t_{c}$ is the duration of the period during which water would be available if all of the water in the soil could be taken up at the constant rate $\bar{\lambda}_{c}$. The flow of water to a plant root from the surrounding soil depends on the geometrical number $\rho_{0}$ and, during the constant rate period, also on the availability number $\tau_{c}$, respectively, defined by

$$
\rho_{0}=r_{0} / r_{1}=\phi_{\mathrm{r}}^{1 / 2}, \quad \tau_{c}=t_{c} / t_{d}=\frac{\widehat{D}\left(\theta_{i}-\theta_{\text {crit }}\right)\left(1-\rho_{o}^{2}\right)}{\bar{\lambda}_{c} r_{1}^{2}} .
$$

The availability number $\tau_{c}$ compares the duration $t_{c}$ of the period water would be available if all of the water in the soil could be taken up at the constant rate $\bar{\lambda}_{c}$ to the characteristic time $t_{d}$ of the capillary flow from the soil to the plant root.

In terms of the dimensionless position $\rho$, time $\tau$, water depletion of the soil $\Theta$, and diffusivity $\Delta$, the flow problem becomes:

flow equation:

$$
\frac{\partial \Theta}{\partial \tau}=\frac{1}{\rho} \frac{\partial}{\partial \rho}\left[\rho \Delta(\Theta) \frac{\partial \Theta}{\partial \rho}\right]
$$

initial condition:

$$
\tau=0, \quad \rho_{0}<\rho<1, \quad \Theta=0 ;
$$

boundary condition at the outer boundary of the cylindrical shell of soil:

$$
\tau>0, \quad \rho=1, \quad \frac{\partial \Theta}{\partial \rho}=0 ;
$$

boundary condition at soil-root interface:

- during the period of constant rate of uptake:

$$
0<\tau<\tau_{\text {crit }}, \quad \rho=\rho_{o}, \quad \Delta(\Theta) \frac{\partial \Theta}{\partial \rho}=\frac{1-\rho_{0}^{2}}{2 \rho_{0} \tau_{c}},
$$

-during the period of falling rate of uptake:

$$
\tau>\tau_{\text {crit }}, \quad \rho=\rho_{o}, \quad \Theta=1 .
$$


From Eq. 52 it follows that the flux at the soil-root interface is large if the product of the geometrical number $\rho_{0}$ and the availability number $\tau_{c}$ is small.

The dimensionless counterpart of Eq. 44, expressing the overall mass balance during the constant rate period, is:

$$
\tau=\tau_{c} \bar{\Theta} .
$$

According to Eq. 54, during the constant rate period the dimensionless time $\tau$ can be replaced by $\tau_{c} \bar{\Theta}$ in all expressions for the distribution of the water content in the cylindrical shell of soil surrounding a root.

\section{Mesoscopic analysis of water uptake by a single root and its macroscopic implications}

\subsection{Approximate solution of linear problem for small geometrical number $\rho_{0}$}

Let us assume that the plant roots are relatively thin and the spatial distribution of the roots is relatively sparse. Then $r_{0} \ll r_{1}$, so that the geometrical number $\rho_{0} \rightarrow 0$, and the solution for a line sink embedded in a soil of infinite extent is an appropriate approximation (Carslaw and Jaeger 1959):

$$
\theta_{i}-\theta=\frac{r_{1}^{2} \bar{\lambda}_{c}}{4 D} \int_{\substack{r^{2} \\ 4 D t}}^{\infty} \frac{\mathrm{e}^{-x}}{x} \mathrm{~d} x=\frac{r_{1}^{2} \bar{\lambda}_{c}}{4 D}\left(-\operatorname{Ei}\left(-\frac{r^{2}}{4 D t}\right)\right)
$$

where $\operatorname{Ei}(. .$.$) is the exponential integral. For small values of r_{1}^{2} /(4 D t)$, and, thus, for large values of $t$, this solution can be approximated by

$$
\theta_{i}-\theta=\frac{r_{1}^{2} \bar{\lambda}_{c}}{4 D}\left(\ln \frac{4 D t}{r^{2}}-\gamma+\frac{4 D t}{r^{2}}+O \frac{4 D t^{2}}{r^{2}}\right) \approx \frac{r_{1}^{2} \bar{\lambda}_{c}}{4 D}\left(\ln \frac{4 D t}{r^{2}}-\gamma\right) .
$$

Philip (1957) used this solution to evaluate the time dependence of the water content at the soil-root interface. Philip was particularly interested in the instant $t=t_{w}$ at which the water content $\theta=\theta_{0}$ at the soil-root interface reaches the value $\theta_{0 w}$ at which the plant wilts. From Eq. 56 at that instant:

$$
\theta_{i}-\theta_{0 w} \approx \frac{r_{1}^{2} \bar{\lambda}_{c}}{4 D}\left(\ln \frac{4 D t_{w}}{r_{0}^{2}}-\gamma\right)
$$

The overall water balance implies that at time $t=t_{w}$ the total volume $u_{c} t_{w}$ taken up is equal to the average depletion $\left(\theta_{i}-\overline{\theta_{w}}\right)$, so that (cf. Eq. 44)

$$
t_{w}=\frac{\left(\theta_{i}-\overline{\theta_{w}}\right)}{\bar{\lambda}_{c}}
$$

where $\phi_{\mathrm{s}} \rightarrow 1$ has been used. Introducing Eq. 58 in Eq. 57 and solving for the average water content $\bar{\theta}$ at wilting gives:

$$
\bar{\theta}_{w}=\theta_{i}-\frac{\mathrm{e}^{\gamma} r_{0}^{2} \bar{\lambda}_{c}}{4 D} \exp \left(\frac{4 D\left(\theta_{i}-\theta_{0 w}\right.}{r_{1}^{2} \bar{\lambda}_{c}}\right) .
$$


The older literature of plant physiology and soil science regarded the water content at which a plant wilted to be a soil property, called the wilting point of a soil. Philip (1957) used Eq. 59 to demonstrate that the average water content at wilting does not only depend on the soil properties $\theta_{0 w}$ and $D$, but also on the geometrical parameters of the plant root system, i.e. the radii $r_{0}$ and $r_{1}$, and the water demand $\bar{\lambda}_{c}$. He concluded that "uncritical use of the "wilting point" as an invariant index of the lower limit of the availability of soil moisture to plants can be very misleading'.

The solution to this problem can be formulated more concisely in terms of dimensionless variables. Corresponding to Eqs. 57-59 we have then:

$$
\begin{gathered}
\Theta \approx \frac{1}{\tau_{c}}\left(\ln \frac{4 \tau}{\rho^{2}}-\gamma\right), \\
\tau_{w}=\tau_{c} \bar{\Theta}, \\
\bar{\Theta}=\frac{\rho_{0}^{2}}{4 \tau_{c}} \exp \left(4 \tau_{c} \Theta_{0 w}+\gamma\right) \quad \text { or } \quad 4 \tau_{c} \bar{\Theta}=\rho_{0}^{2} \exp \left(4 \tau_{c} \Theta_{0 w}+\gamma\right) .
\end{gathered}
$$

4.2 Transient soil water depletion regarded as a succession of steady-state profiles

Gardner (1960) used the two-term approximation of (56) to calculate the water depletion pattern around individual roots. But perhaps more importantly, he also used it as a point of departure for the formulation of a simpler model, in which the depletion resulting from uptake by a single root is treated as a series of steady flows in the cylindrical shell of soil surrounding the root, with the soil-root interface at the inner edge and the water coming from the outer edge. This simple model has served ever since as a point of departure for more sophisticated mesoscopic as well as macroscopic models of water uptake.

In the single root model of Gardner (1960), the root is viewed as a cylinder of uniform radius $r_{0}$ and infinite length having uniform water absorbing properties. For steady state conditions $\partial \theta / \partial t=0$ in the soil shell surrounding the root with water flowing from the outer cylindrical surface at $r=r_{1}$ to the inner cylindrical soil-root interface at $r=r_{0}$, the solution of Eq. 2 under the assumption of constant $k$ gives the following expression for the flux at the soil-root interface at the meso-scale:

$$
(\theta v)_{\mathrm{r}}=G k\left(h_{1}-h_{0}\right),
$$

where $(\theta v)_{\mathrm{r}}$ is the rate of water uptake per unit length of root, $h_{1}$ is pressure head of the soil water at $r=r_{1}, h_{0}$ is the pressure head at the soil-root interface, and the dimensionless geometric and root distribution factor $G$ is given by

$$
G=\frac{2 \pi}{\ln \left(r_{1} / r_{0}\right)} .
$$

At the macro-scale, the volumetric rate of uptake $\bar{\lambda}$ is obtained by multiplying both sides of (63) by the root length density $L$ :

$$
\bar{\lambda}=G L k\left(h_{1}-h_{0}\right) .
$$

Cowan (1965) realized that the assumption of constant $k$ used in deriving (65) can be avoided. In essence he replaced (65) by

$$
\bar{\lambda}=G L k_{\mathrm{av}}\left(h_{1}-h_{0}\right)=G L D_{\mathrm{av}}\left(\theta_{1}-\theta_{0}\right)=G L\left(\phi_{1}-\phi_{0}\right),
$$


where the average hydraulic conductivity $k_{\mathrm{av}}$ and average diffusivity $D_{\mathrm{av}}$ are defined by

$$
k_{\mathrm{av}}=\frac{\int_{h_{0}}^{h_{1}} k \mathrm{dh}}{h_{1}-h_{0}}, \quad D_{\mathrm{av}}=\frac{\int_{\theta_{0}}^{\theta_{1}} D \mathrm{~d} \theta}{\theta_{1}-\theta_{0}} .
$$

The integrals in (67) can be easily evaluated for any of the commonly used expressions to represent the $k(h)$ and $D(\theta)$ relationships for specific classes of soils.

In an attempt to account for the location of the soil layer, one might be tempted to replace $h_{0}$ in (65) and (66) by $h_{\text {rootsystem }}-z$, where $h_{\text {rootsystem }}$ is the pressure head in the root system evaluated at the soil surface. However, as will become apparent from an analysis of the hydraulic connection between the soil-root interface and the xylem discussed later on, this cannot be justified.

\subsection{Period of constant rate of uptake}

\subsubsection{Solution of linear problem}

The solution of Eq. 49 subject to Eqs. $50-52$ is given by De Willigen and Van Noordwijk (1987):

$$
\begin{aligned}
\tau_{c} \Theta= & \tau+\frac{\rho^{2}}{4}-\frac{1}{2} \ln \rho-\frac{3+4 \rho_{0}^{2} \ln \rho_{0}-2 \rho_{0}^{2}-\rho_{0}^{4}}{8\left(1-\rho_{0}^{2}\right)} \\
& -\sum_{n=1}^{\infty} A_{n}\left(\rho, \rho_{0}\right) \exp \left(-\beta_{n}^{2} t\right)
\end{aligned}
$$

with

$$
A_{n}\left(\rho, \rho_{0}\right)=\frac{\pi\left(1-\rho_{0}^{2}\right)}{2} \frac{J_{1}^{2}\left(\beta_{n}\right)\left(J_{0}\left(\beta_{n} \rho\right) Y_{1}\left(\beta_{n} \rho_{0}\right)-Y_{0}\left(\beta_{n} \rho\right) J_{1}\left(\beta_{n} \rho_{0}\right)\right)}{\beta_{n} \rho_{0}\left(J_{1}^{2}\left(\beta_{n} \rho_{0}\right)-J_{1}^{2}\left(\beta_{n}\right)\right)},
$$

and the $\beta_{n}$ are the roots of

$$
J_{1}\left(\beta_{n} r_{0}\right) Y_{1}\left(\beta_{n}\right)-J_{1}\left(\beta_{n} Y_{1}\left(\beta_{n} r_{0}\right)=0 .\right.
$$

Equation describes the distribution of the water content around an individual root resulting from a constant rate of uptake. The structure of this solution is very simple. Two parts can be distinguished:

- terms guaranteeing that the initial condition is satisfied: they have the form of an exponentially fading, infinite series with a characteristic time $\tau_{d}$;

- terms guaranteeing that the flux boundary conditions and the overall mass balance are satisfied: they have the form of a profile of time invariant form, with the water content decreasing linearly with time everywhere at the same rate.

Using Eq. 54, expressing the proportionality between $\tau$ and $\Theta$ in the constant rate phase, Eq. 68 can also be written as:

$$
\begin{aligned}
\tau_{c} \Theta= & \tau_{c} \bar{\Theta}+\frac{\rho^{2}}{4}-\frac{1}{2} \ln \rho-\frac{3+4 \rho_{0}^{2} \ln \rho_{0}-2 \rho_{0}^{2}-\rho_{0}^{4}}{8\left(1-\rho_{0}^{2}\right)} \\
& -\sum_{n=1}^{\infty} A_{n}\left(\rho, \rho_{0}\right) \exp \left(-\beta_{n}^{2} \tau_{c} \bar{\Theta}\right) .
\end{aligned}
$$


In the soil science-plant physiology literature the asymptotic, time invariant profiles are usually referred to as steady rate profiles (Cowan 1965; Passioura and Cowan 1968). Distributions of water content of time invariant form have also been postulated and calculated for the more complicated mixed boundary condition at the soil root interface with the flux being zero over part of this interface due to poor contact between the plant and the soil (De Willigen and Van Noordwijk 1987).

\subsubsection{Transient depletion as a succession of steady rate profiles}

An attractive alternative to (65) or (66) is to regard the withdrawal of water to be uniformly distributed over the cylindrical shell of soil. In fact, if Richards equation were linear and the water demand were constant, there would be a solid basis for this at intermediate times when the memory of the details of the initial water content distribution has already faded and the constant demand can still be met.

Assuming steady rate conditions, i.e. $\partial \theta / \partial t=$ constant, and zero flux at the outer cylindrical surface at $r=r_{1}$ of the soil shell surrounding the root, the solution of (1) and (2) for the meso-scopic flux $(\theta v)_{\mathrm{r}}$ at the soil-root interface is (Passioura and Cowan 1968; Jacobsen 1974):

$$
(\theta v)_{\mathrm{r}}=G^{*} k\left(h_{1}-h_{2}\right)=G^{*} D\left(\theta_{1}-\theta_{0}\right)=G^{*}\left(\phi_{1}-\phi_{0}\right),
$$

where the dimensionless geometric and root distribution factor $G^{*}$ is given by

$$
G^{*}=\frac{2 \pi}{\left[1-\left(r_{0} / r_{1}\right)\right]^{-1} \ln \left(r_{1} / r_{0}\right)-1 / 2} .
$$

For $r_{0} \ll r_{1}$, the factor $G^{*}$ can be approximated by

$$
G^{*} \approx \frac{2 \pi}{\ln \left(r_{1} / r_{0}\right)-1 / 2} \approx \frac{2 \pi}{\ln \left(0.6064 r_{1} / r_{0}\right)} .
$$

Comparison of the expressions for $G$ in (64) and for $G^{*}$ in (74) shows that whereas in the steady state approximation all the water is assumed to originate from $r=r_{1}$, in the steady rate approximation it seemingly originates from $r=0.6064 r_{1}$.

The corresponding steady rate mesoscopic expression for the volumetric rate of uptake $\bar{\lambda}$ is:

$$
\bar{\lambda}=G^{*} L k\left(h_{1}-h_{0}\right)
$$

The steady rate feature has long been known as an important term in the full solutions of linear equations subject to flux boundary conditions for heat conduction and flow to wells. Cowan (1965) demonstrated its relevance for flow to plant roots, even for a diurnally varying, but on average constant demand. Passioura and Cowan (1968) compared the predictions of the water content at the soil root interface based on the approximate steady state and steady rate solutions and the exact numerical solution, finding reasonable agreement among them, with the steady rate method being somewhat more accurate than the steady state one. 


\subsection{Period of falling rate of uptake}

\subsubsection{Linear problem}

Of course, the water content cannot decrease indefinitely. At a certain instant $t_{\text {crit }}$, the water content at the soil-root interface reaches a critical value $\theta_{w 0}$ and then the flux boundary condition at the soil-root interface will switch to a water content boundary condition. From that time onwards the rate of supply of water from the soil will govern the falling rate of uptake. If the Richards equation were linear, in the falling rate phase the solution would again be the sum of two parts:

- an exponentially fading infinite series with characteristic diffusion time $t_{d}=r_{1}^{2} / D$, which guarantees that the intermediate condition at the transition from the constant rate phase to the falling rate phase is satisfied;

- a term that has the form of the product of two terms, one depending on the radial coordinate and one depending exponentially on time, again involving the characteristic diffusion time $t_{d}=r_{1}^{2} / D$,

The change from the constant rate phase to the falling rate phase is basically a change from the plant-dominated boundary condition to the soil-dominated boundary condition. Constant and falling rates also occur for the uptake of nutrients by plant roots (De Willigen and Van Noordwijk 1994a,b). Similar switches of boundary conditions are well known in other environmental physics problems. Evaporation from saturated bare soil, starting at a constant rate being determined only by atmospheric conditions, may at a certain instant switch to a falling rate phase in which the evaporation rate depends only on the hydraulic properties of the soil. For infiltration of water into soil, an analogous switch occurs at the so-called ponding time.

\subsubsection{Nonlinear problem}

Introducing Eq. 7 in Eq. 49, using the definitions of $\Theta$ and $\Delta$ in (45) gives:

$$
\frac{\partial(b-\Theta)}{\partial \tau}=\frac{\partial}{\partial \rho^{2}} 4 a \rho^{2}(b-\Theta)^{n} \frac{\partial(b-\Theta)}{\partial \rho^{2}},
$$

where

$$
a=\frac{(n+1)\left(\theta_{i}-\theta_{\text {crit }}\right)^{n+1}}{\theta_{i}^{n+1}-\theta_{\text {crit }}^{n+1}}, \quad b=\frac{\theta_{i}}{\theta_{i}-\theta_{\text {crit }}} .
$$

In terms of the dependent variable $b-\Theta$, the boundary condition (53) at the soil-root interface for the falling rate phase reduces to

$$
\tau>\tau_{\text {crit }}, \quad \rho=\rho_{o}, \quad b-\Theta=b-1 .
$$

For the special case with $\theta_{\text {crit }}=0$, so that $b=1$ in (76-78), a possible solution of Eq. 76 subject to (78) has the form of a product of two terms, one depending on $\rho$ and the other depending on $\tau$ (cf., Aronson and Peletier 1981):

$$
(1-\Theta(\tau, \rho))=\left(1-\Theta_{\tau}(\tau)\right)\left(1-\Theta_{\rho}(\rho)\right) .
$$


Introducing Eq 79 in the 'porous medium equation' (76) with $b=1$ gives two ordinary differential equations, one in $\tau$ and the other in $\rho$ :

$$
\begin{gathered}
\frac{\mathrm{d}\left(1-\Theta_{\tau}\right)}{\mathrm{d} \tau}=\lambda\left(1-\Theta_{\tau}\right)^{n+1}, \\
\frac{\mathrm{d}}{\mathrm{d} \rho^{2}}\left(4 a\left(1-\Theta_{\rho}\right)^{n} \rho^{2} \frac{\mathrm{d}\left(1-\Theta_{\rho}\right)}{\mathrm{d} \rho^{2}}\right)=\lambda\left(1-\Theta_{\rho}\right) .
\end{gathered}
$$

The solution of the ordinary differential equation (80) in $\tau$ is:

$$
1-\Theta_{\tau}(\tau)=\left(\left(1-\Theta_{\tau}\left(\tau_{0}\right)\right)^{-n}-n \lambda\left(\tau-\tau_{o}\right)\right)^{-1 / n} .
$$

A solution of the nonlinear ordinary differential equation (81) in $\rho$ for this cylindrically symmetric problem is not known to me. Even so, an interesting expression for $1-\Theta$ at time $\tau$ is obtained by introducing Eq. 82 in Eq. (79):

$$
1-\Theta(\tau, \rho)=\left(\left(1-\Theta_{\tau}\left(\tau_{0}\right)\right)^{-n}-n \lambda\left(\tau-\tau_{o}\right)\right)^{-1 / n}\left(1-\Theta_{\rho}(\rho)\right) .
$$

On the right hand side of Eq. 83, bringing $\left(1-\Theta_{\rho}(\rho)\right)$ inside the parenthesis of the $\tau$-dependent term and noting that

$$
1-\Theta\left(\tau_{0}, \rho\right)=\left(1-\Theta_{\tau}\left(\tau_{0}\right)\right)\left(1-\Theta_{\rho}(\rho)\right),
$$

gives:

$$
1-\Theta(\tau, \rho)=\left(\left(1-\Theta\left(\tau_{0}, \rho\right)\right)^{-n}-n \lambda\left(1-\Theta_{\rho}\right)^{-n}\left(\tau-\tau_{0}\right)\right)^{-1 / n} .
$$

Also without knowing the solution for $1-\Theta_{\rho}$, we can write for the average water content at time $\tau$ :

$$
1-\bar{\Theta}(\tau)=\left(1-\Theta_{\tau}(\tau)\right)\left(1-\bar{\Theta}_{\rho}\right) .
$$

From Eqs. 86 and 82 it follows that the average water content $\bar{\Theta}$ satisfies an equation of the same form as Eq. 85:

$$
1-\bar{\Theta}(\tau)=\left(\left(1-\bar{\Theta}\left(\tau_{0}\right)\right)^{-n}-n \lambda\left(1-\bar{\Theta}_{\rho}\right)^{-n}\left(\tau-\tau_{0}\right)\right)^{-1 / n} .
$$

This nonlinear relationship between $\bar{\Theta}$ and $\tau$ for the falling rate period can be seen as the counterpart of the simple linear relationship (54) between these two variables in the constant rate period. Such explicit expressions for the average water content are a promising basis for upscaling from the mesoscopic to the macroscopic scale.

\subsection{Hydraulic connection between the soil-root interface and the xylem}

Taylor and Klepper (1975) used a steady state model (cf. Section 4.2) to interpret experimental data for uptake of water by cotton root systems. Specifically, they used the following equations of the same form as (65) and (66):

$$
\bar{\lambda}=B_{\text {soil-rootsystem }} L k_{\text {soil-rootsystem }}\left(h_{1}-h_{\text {xylem }}\right),
$$

where $h_{\text {xylem }}$ is a value obtained from shoot water potential measurements, $k_{\text {soil }}-$ rootsystem is the hydraulic conductivity of the combined soil-root pathway, and the dimensionless geometric and root distribution factor $B_{\text {soil-rootsystem }}$ is given by

$$
B_{\text {soil-rootsystem }}=\frac{2 \pi}{\ln \left(r_{1} / r_{\text {stele }}\right)},
$$


where $r_{\text {stele }}$ is the radius of the root stele. Taylor and Klepper (1975) demonstrated that in the wet range $k_{\text {soil-rootsystem }}$ was up to six orders of magnitude smaller than $k(\theta)$ for just the soil. This strongly suggests that, at least for cotton, most of the resistance resides in the roots, except when the soil is very dry and $k_{\text {soil-rootsystem }}$ becomes comparable to $k_{\text {av }}$ given by (67).

Herkelrath et al. (1977a) investigated the influence of $\theta$ and $h$ upon root water uptake in a laboratory experiment with winter wheat. They used wax layers, penetrable by roots but not by water, to divide their soil column in different sections. Their observations imply a large resistance between the soil-root interface and the xylem and a small resistance between the xylem and the leaves. Water uptake began to decrease rapidly when $\theta$ fell below about 0.10 , corresponding to about $h=-0.1$ bar, in agreement with the data of Taylor and Klepper for cotton.

To account for their observations, Herkelrath et al. (1977b) complemented the steady state model (63) or the steady rate model (72) with the following expression to describe the flow from the soil-root interface to the xylem:

$$
q_{\mathrm{r}}=C \frac{\theta_{0}}{\theta_{\mathrm{s}}}\left(h_{0}-h_{\mathrm{xylem}}\right),
$$

where $C$ is the conductance of the region between the soil and the xylem. The degree of saturation $\theta_{0} / \theta_{\mathrm{s}}$ of the soil at the soil/root interface is a factor accounting for the poor contact between the soil and the root. In view of the $\theta_{0}$ proportionality in (90), it is interesting to note that for $\theta>0.03$, Taylor and Klepper (1975) found a more or less linear increase of the rate of uptake with $\theta$. Eq.(90) is similar to an equation commonly used to describe the steady flow of water across a surface crust into the underlying soil where the water is at a uniform water content and pressure head and steadily flows downward under the influence of gravity. Raats (1974b) described a simple graphical procedure to analyze such steady infiltration into a crusted soil. Van Noordwijk (1983), see also De Willigen and Van Noordwijk (1987) and Raats (1990a), used a similar procedure to analyze the implications of (90). For a critical value of $h_{\text {xylem }}=h_{\text {xylemw }}$ at which wilting starts, it follows from (90) that:

$$
h_{0 w}=h_{0 \mathrm{xylemw}}+\frac{\theta_{\mathrm{s}}}{\theta_{0 w}} \frac{q_{\mathrm{r}}}{C} .
$$

The graphical or equivalent iterative procedure consists of reducing the seemingly infinity of possible pairs to a single pair by determining the intersection of (91) with the soil water retention curve:

$$
h_{0 w}=h\left(\theta_{0 w}\right) .
$$

The root contact model (90) has been used extensively by Jensen et al $(1990,1993)$. The concept of contact resistance is particularly relevant for heavier textured soils in which the roots have a strong tendency to grow at the surface of aggregates.

In view of (72), (75) and (90) the flow from the soil to the xylem can be described by

$$
\bar{\lambda}=B^{*} k L\left(h_{1}-h_{0}\right)=C \frac{\theta_{0}}{\theta_{\mathrm{s}}} L\left(h_{0}-h_{\mathrm{xylem}}\right) .
$$

Assuming that he soil conductance is large in comparison with the root conductance, i.e. $B^{*} k L\left(h_{1}-h_{0}\right) \gg C L \theta_{0} / \theta_{\mathrm{s}}$ or $B^{*} k\left(h_{1}-h_{0}\right) \gg C \theta_{0} / \theta_{\mathrm{s}}$, so that the water status around the individual roots is nearly uniform, i.e. $h_{0} \approx \bar{h} \approx h_{1}$ and $\theta_{0} \approx \bar{\theta} \approx \theta_{1}$, 
where $\bar{h}$ is the pressure head and $\bar{\theta}$ is the water content at the macroscopic scale. Equation 93 may hen be replaced by

$$
\bar{\lambda}=C \frac{\bar{\theta}}{\theta_{\mathrm{s}}} L\left(\bar{h}-h_{\mathrm{xylem}}\right) .
$$

If in addition the xylem conductance is large in comparison with the root conductance $C L \theta_{0} / \theta_{\mathrm{s}}$, so that the xylem pressure head $h_{\text {xylem }}$ at depth $z$ can be written as the sum of the root pressure head $h_{\text {root }}$ evaluated at the soil surface and the gravitational head $z$, i.e. $h_{\text {xylem }}=h_{\text {root }}-z$, then (94) further reduces to

$$
\bar{\lambda}=C \frac{\bar{\theta}}{\theta_{\mathrm{s}}} L\left(\bar{h}-h_{\text {root }}+z\right) .
$$

Clearly, Eq. 95 should only be used if the soil resistance and the xylem resistance are negligible.

\section{Macroscopic models for water uptake}

5.1 Macroscopic physical models directly inspired by mesoscopic models

In Sect. 4 it was shown that mesoscopic analysis of water uptake by a single root based on a variety of assumptions can be used to infer various forms of the macroscopic volumetric rate of uptake $\bar{\lambda}$.

Philip (1957) used Eq. 59 to cast doubt on the concept of the "wilting point" as an invariant index of the lower limit of the availability of soil water to plants. Following this pioneering study, Philip (personal communication, sometime in the 1980s) in later years became rather skeptical about models at the scale of individual roots. In fact, when he returned to the problem of uptake of water by plants later (Philip 1991, 1997), he introduced the root extraction rate directly as a sink term in the flow equation, recognizing, of course, that it depends on the water status of the soil, the characteristics of the soil and the root system, and the meteorological conditions.

Equation 65, based on the transient water depletion regarded as a succession of steady states by Gardner (1960), has been widely used to interpret uptake data. An example is the analysis by Feddes (1971) of uptake of water by cabbage from a clay soil. In Sect. 4 several variants of the Gardner model were also discussed: the generalization by Cowan (1965) leading to (66), the steady rate version (75) of Passioura and Cowan (1968) and Jacobsen (1974), the identification in (88) of the resistance residing in the region between the soil-root interface and the xylem by Taylor and Klepper (1975), and the root contact model (93) of Herkelrath et al. (1977a,b). Despite all this attention, like Philip, Gardner (1991) more recently also became rather skeptical about upscaling from the mesoscopic to the macroscopic scale. He specifically pleaded for a more direct semi-empirical, macroscopic approach, with due attention for the effect of solutes on water uptake, as was pioneered at the mesoscopic scale by Dalton et al. (1975) and by Fiscus (1975).

The steady rate approximation has been extensively used by De Willigen and Van Noordwijk (1987), De Willigen et al. (2000), Heinen (1997), and Heinen and De Willigen $(1998,2001)$, not only for uptake of water, but also for the uptake of nutrients 
(De Willigen and Van Noordwijk 1994a,b). They have also considered extensions to cases where the flow to the individual roots is not radially symmetric, either due to partial soil-root contact or due to non-uniform spatial root distributions. De Willigen and Van Noordwijk (1987,Chapter 10) demonstrated that the fraction of the potentially available water that can be acquired by the root system at a certain rate decreases with decreasing soil-root contact and increasing spatial clustering of roots. In effect this is an alternative to the method for dealing with limited soil-root contact discussed in Sect. 4.5. Whitaker 1986 (1992) showed that limited soil-root contact leads to a decrease of uptake of water and nitrate, explaining the existence of an optimum in the response curve of crops to soil compaction.

Yet another approach was explored tentatively by Rappoldt (1992), as a byproduct of a practical method for the description of transport processes at the mesoscopic scale in aggregated soils. His method is based on a simplification of the complex geometry of natural structural soils, in such a manner that the essential geometric and structural characteristics governing diffusion processes are preserved. This is done as follows. The geometry of the natural system is replaced by a model system of plates, or cylinders, or spheres. The distribution of the thicknesses of the plates, or the radii of the cylinders or spheres, is chosen such that the structured soil and the model system have the same distance distribution. Calculations of diffusion processes are carried out in the model system. On this basis, Rappoldt (1992) analyzed in detail various aspects of aeration of heterogeneous soils (see also Rappoldt 1990; Rappoldt and Verhagen 1999). To illustrate the generality of the method, he also used it to analyze the uptake of water and nutrients by an irregularly distributed root system (Rappoldt 1992, chapter 7). The full treatment of this uptake model remains to be published.

5.2 Macroscopic empirical models indirectly inspired by mesoscopic models

Denmead and Shaw (1962) experimentally verified the early predictions that water is not equally available to plants in the range between field capacity and the permanent wilting point, and that transpiration can be restricted and plants can wilt over a wide range of soil water contents, depending on root density, on the soil hydraulic properties, and last but not least on the transpiration demand of the atmosphere. Tanner (1967, Fig. 5) nicely summarized other data available at that time relating the ratio of actual and potential transpiration to the amount of water in the soil.

Based on such data, observed spatial-temporal patterns of the volumetric water uptake, and the results of the mesoscopic approach, Feddes et al. (1978) boldly proposed a volumetric rate of uptake of the form:

$$
\bar{\lambda}\left(h, T_{p}\right)=\alpha\left(h, T_{p}\right) \bar{\lambda}_{p},
$$

where $\alpha\left(h, T_{p}\right)$ is a dimensionless, prescribed function of the pressure head and the potential transpiration rate $T_{p}$ governed by atmospheric conditions, and $\bar{\lambda}_{p}$ is the potential, i.e. maximal possible, rate of uptake. Under optimal water conditions, $\bar{\lambda}_{p}$ integrated over the rooting depth is equal to $T_{p}$. For the reduction function $\alpha\left(h, T_{p}\right)$ Feddes et al. (1978) proposed: 


$$
\alpha\left(h, T_{p}\right)=\begin{array}{rlrl}
0 & & \geq h_{1} \\
\frac{h_{1}-h}{h_{1}-h_{2}} & h_{1}>h & \geq h_{2} \\
1 & h_{2}>h & \geq h_{3}\left(T_{p}\right), \\
\frac{h-h_{4}}{h_{3}\left(T_{p}\right)-h_{4}} h_{3}\left(T_{p}\right) & >h \geq h_{4} \\
0 & h_{4} & >h
\end{array}
$$

where $h_{1}$ is the pressure head above which the water uptake is zero due to oxygen deficiency, $h_{4}$ is the pressure head below which the water uptake is zero due to dryness, and $h_{2}$ and $h_{3}$ are pressure heads between which the water uptake is maximal. Between $h_{1}$ and $h_{2}$ and between $h_{3}$ and $h_{4}$ a linear variation is assumed. In line with the data of Denmead and Shaw (1962), the value of $h_{3}$ depends on the potential transpiration $T_{p}$.

Van Genuchten (1987) proposed an extension of the macroscopic empirical model to include reduction of water uptake due to osmotic stress. He replaced the piecewise linear water stress reduction function (97) of Feddes et al. (1978) by a nonlinear reduction function $\alpha(h)$, introduced a similar osmotic stress reduction function $\alpha\left(h_{o}\right)$, where $h_{o}$ is the osmotic pressure, and explored additive and multiplicative combinations of these as net water-salinity stress reduction functions. Feddes and Raats (2004) reviewed the rather extensive literature that grew out of this proposal. It appears that such semi-empirical approaches are now favored in simulation models. Of course, in following this trend, one should try not to not loose sight of the physical and physiological basis of the phenomena.

Acknowledgements An early version of this paper was presented at the conference Multiscale Problems in Science and Technology. Challenges to Mathematical Analysis and Perspectives, organized by the Department of Mathematics, University of Zagreb, held 4-10 September 2000 held at the InterUniversity Center (IUC) at Dubrovnik, Croatia. The present version owes much to discussions with Prof Reinder A. Feddes of Wageningen University during the preparation of a recent review paper on uptake of water (Feddes and Raats 2004). In particular, he brought to my attention the work discussed and interpreted in Sect. 2.2 on the power law model of Gale and Grigal and the exponential model of Arora and Boer.

\section{References}

Aronson, D.G., Peletier, L.A.: Large time behaviour of solutions of the porous medium equation in bounded domains. J. Differ. Equ. 39, 378-412 (1981).

Arora, V.K., Boer, G.J.: A representation of variable root distribution in dynamic vegetation models. Earth Interact. 7, Paper No. 6, 1-19 (2003)

Carslaw, H.S., Jaeger J.C.: Conduction of Heat in Solids, 2nd edn. Oxford University Press, London (1959)

Cowan, I.R.: Transport of water in the soil-plant-atmosphere system. J. Appl. Ecol. 2, 221-239 (1965)

Dalton, F.N., Raats, P.A.C., Gardner, W.R.: Simultaneous uptake of water and solutes by plant roots. Agronomy J. 67, 334-339 (1975)

Denmead, O.T., Shaw, R.H.: Availability of soil water to plants as affected by soil moisture content and meteorological conditions. Agron. J. 54, 385-390 (1962)

De Willigen, P., Van Noordwijk, M.: Roots, plant production and nutrient use efficiency. PhD Thesis, Wageningen Agricultural University (1987)

De Willigen, P., Van Noordwijk, M.: Mass flow and diffusion of nutrients to a root with constant or zero-sink uptake 1. Constant uptake. Soil Sci. 157, 162-170 (1994a)

De Willigen, P., Van Noordwijk, M.: Mass flow and diffusion of nutrients to a root with constant or zero-sink uptake 2. Zero-sink uptake. Soil Sci. 157, 171-175 (1994b) 
De Willigen, P., Nielsen, N.E., Claassen, N., Castrignan, A.M.: Modelling water and nutrient uptake. In: Smit A.L., Bengough G., Engels C., van Noordwijk M., Pellerin S., van de Geijn S. (eds.) Root Methods: A Handbook. Springer, Berlin (2000)

Feddes, R.A.: Water, heat and crop growth. PhD Thesis, Wageningen Agricultural University (1971)

Feddes, R.A., Kowalik, P.J., Zaradny, H.: Simulation of Field Water Use and Crop Yield. Simulation Monograph, Pudoc, Wageningen (1978)

Feddes, R.A., Hoff, H., Bruen, M., Dawson, T., de Rosnay, P., Dirmeyer, P., Jackson, R.B., Kabat, P., Kleidon, A., Lilly, A., Pitman, A.J.: Modeling root water uptake in hydrological and climate models. Bull. Am. Meteorol. Soc. 82, 2797-2809 (2001)

Feddes, R.A., Raats, P.A.C.: Parameterizing the soil-water-plant root system, In: Feddes R.A., De Rooij G.H., Van Dam J.C. (eds.) Wageningen UR Frontis Series, vol. 6, Unsaturated-zone Modeling, Progress, Challenges and Applications, pp. 95-141. Kluwer, Dordrecht (2004)

Fiscus, E.L.: The interaction between osmotic and pressure-induced water flow in plant roots. Plant Physiol. 55, 917-922 (1975).

Gale, M.R., Grigal, D.F.: Vertical root distributions of northern tree species in relation to successional status. Can. J. Forestry Res. 17, 829-834 (1987)

Gardner, W.R.: Dynamic aspects of water availability to plants. Soil Sci. 89, 63-73 (1960)

Gardner, W.R.: Modeling water uptake by roots. Irrigat. Sci. 12, 109-114 (1991)

Gerwitz, A., Page, E.R.: An empirical mathematical model to describe plant root systems. J. Appl. Ecol. 11, 773-781 (1974)

Gilding, B.H.: Qualitative mathematical analysis of the Richards equation. Transport Porous Media 5, 561-566 (1991)

Heinen, M.: Dynamics of water and nutrients in closed, recirculating cropping systems in glasshouse horticulture: with special attention to lettuce grown in irrigated sand beds. PhD Thesis, Wageningen Agricultural University (1997)

Heinen, M., De Willigen, P.: FUSSIM2: A two-dimensional simulation model for water flow, solute transport, and root uptake of water and nutrients in partly unsaturated porous media. In: Quantitative Approaches in Systems Analysis, vol. 20. DLO Research Institute for Agrobiology and Soil Fertility, Wageningen (1998)

Heinen, M., De Willigen, P.: FUSSIM2: New features and updated user's guide. Alterra Report, vol. 363. Alterra, Wageningen (2001)

Herkelrath, W.N., Miller, E.E., Gardner, W.R.: Water uptake by plants I Divided root systems. Soil Sci. Soc. Am. J. 41, 1033-1037 (1977a)

Herkelrath, W.N., Miller, E.E., Gardner, W.R.: Water uptake by plants II The root contact model. Soil Sci. Soc. Am. J. 41, 1033-1037 (1977b)

Huxley, J.S.: Problems of Relative Growth, 2nd edn. Republication of 1932 1st edition published by Methuen, London, with a new introduction by the author and a supplementary essay by E.C.R. Reeve and the author. Dover, New York (1972)

Jackson, R.B.: The importance of root distributions for hydrology, biogeochemistry, and ecosystem functioning. In: Tenhunen J.D., Kabat P. (eds.) Integrating Hydrology, Ecosystem Dynamics, and Biogeochemistry in Complex Landscapes, Report of the Dahlem Workshop, Berlin, January 18-23, 1998, Wiley, Chichester, UK (1999)

Jackson, R.B., Canadell, J., Ehleringer, J.R., Mooney, H.A., Sala, O.E., Schulze, E.D.: A global analysis of root distributions for terrestrial biomes. Oecologia 108, 389-411 (1996)

Jackson, R.B., Mooney, H.A., Schulze, E.D.: A global budget for fine root biomass, surface area, and nutrient contents. Proc. Natl. Acad. Sci. USA 94, 7362-7366 (1997)

Jacobsen, B.F.: Water and phosphate transport to plant roots. Acta Agricult. Scand. 24, 55-60 (1974)

Jensen, C.R., Hensen, I.E., Hansen, S.: A root contact model and potential differences to water flow in the soil plant system. In: Sinha S.K., Sane P.V., Bhargava S.C., Agrawal P.V. (eds.) Proceedings of the International Congress of Plant Physiology. New Delhi, India, February 15-20, 1988, vol. 2, pp. 825-831. Society for Plant Physiology and Biochemistry, New Delhi (1990)

Jensen, C.R., Svendsen, H., Andersen, M.N., Lösch, R.: Use of the root contact concept, an empirical leaf conductance model and pressure-volume curves in simulating crop water relations. Plant Soil 149, 1-26 (1993)

Kirchhoff, G.: Vorlesungen über der Theorie der Wärme. Barth, Leipzig (1894)

Miller, E.E., Miller, R.D.: Physical theory of capillary flow phenomena. Physics 27, 324-332 (1956)

Passioura, J.B., Cowan, I.R.: On solving the non-linear diffusion equation for the radial flow of water to roots. Agricult. Metorol. 5, 129-134 (1968)

Philip, J.R.: The physical principles of soil water movement during the irrigation cycle. In: Proc. Intern. Congr. Irrigation and Drainage, 3rd, San Francisco, vol. 8, pp. 125-153. (1957) 
Philip, J.R.: Effects of root and subirrigation depth on evaporation and percolation losses. Soil Sci. Soc. Am. J. 55, 1520-1523 (1991)

Philip, J.R.: Effect of root water extraction on wetted regions from continuous irrigation sources. Irrigat. Sci. 17, 127-135 (1997)

Raats, P.A.C.: Steady infiltration from line sources and furrows. Soil Sci. Soc. Am. Proc. 34, 709-714 (1970)

Raats, P.A.C.: Steady flows of water and salts in uniform soil profiles with plant roots. Soil Sci. Soc. Am. Proc. 38, 717-722 (1974a)

Raats, P.A.C.: Steady infiltration into crusted soils. 10th Int. Congress Soil Sci. (Moscow) Trans. 1, 75-80 (1974b)

Raats, P.A.C.: The distribution of the uptake of water by plants: inference from hydraulic and salinity data. In: Seminaires sur l'irrigation localisée. 1. Mouvement de l'eau et des sels en function des charactéristiques des sols soumis à l'irrigation localisée, Proc. AGRIMED Seminar on the Movement of Water and Salts as a Function of the Properties of the Soil under Localized Irrigation, held 6-9 November 1979 at Bologna, Italy, pp. 35-46. Institut d'Agronomie de l'Université de Bologne, Bologna, Italy, (1982)

Raats, P.A.C.: Applications of the theory of mixtures in soil science. In: Truesdell C. (ed.) Rational Thermodynamics, with an Appendix by C.-C. Wang, 2nd edn, corrected and enlarged, to which are adjoined appendices by 23 authors. Chapt Appendix 5D, pp. 326-343. Springer Verlag, New York (1984)

Raats, P.A.C.: Characteristic lengths and times associated with processes in the root zone. In: Hillel D., Elrick D.E. (eds.) Scaling in Soil Physics: Principles and Applications, pp. 59-72. Soil Science Society of America, Madison, Wisconsin, USA (1990a)

Raats, P.A.C.: On the roles of characteristic lengths and times in soil physical processes. In: Proc. 14th Int. Congr. Soil Sci., held 12-18 August 1990, at Kyoto, Japan, vol. 1, pp. 202-207 (1990b)

Raats, P.A.C.: A superclass of soils. In: Van Genuchten M.Th., Leij F.J., Lund L.J. (eds.) Indirect Methods for Estimating the Hydraulic Properties of Unsaturated Soils, Proceedings of an International Workshop, held 11-13 Oct. 1989 at Riverside, Calif., USA, Univ. of Calif., Riverside, pp. 49-51 (1992)

Raats, P.A.C.: Spatial and material description of some processes in rigid and non-rigid saturated and unsaturated soils. In: Thimus J.-F., Abousleiman Y., Cheng, A.H.-D., Coussy O., Detournay E. (eds.) Poromechanics - A tribute to Maurice A. Biot, Proceedings of the Biot Conference on Poromechanics, held September 14-16, 1998 at Louvain-la-Neuve, pp. 135-140. Belgium, Balkema, Rotterdam, The Netherlands (1998)

Raats, P.A.C.: Developments in soil-water physics since the mid 1960s. Geoderma 100, 355-387 (2001)

Raats, P.A.C., Smiles, D.E., Warrick, A.W.: Contributions to environmental mechanics: Introduction. In: Raats P.A.C., Smiles D.E., Warrick A.W. (eds.) Geophysical Monograph 129, Environmental Mechanics: Water, Mass and Energy Transfer in the Biosphere, pp. 1-28. American Geophysical Union, Washington, DC (2002)

Rappoldt, C.: The application of diffusion models to an aggregated soil. Soil Sci. 150, 645-661 (1990)

Rappoldt, C.: Diffusion in aggregated soil. PhD Thesis, Wageningen Agricultural University (1992)

Rappoldt, C., Verhagen, J.H.G.: Equivalent cylinder systems representing the soil matrix in diffusionreaction models for an aggregated soil. Transport Porous Media 37, 1-24 (1999)

Richards, L.A.: Capillary conduction of liquids through porous mediums. Physics 1, 318-333 (1931)

Tanner, C.B.: Measurement of evapotranspiration. Agronomy 11, 534-574 (1967)

Taylor, H.M., Klepper, B.: Water uptake by cotton root systems: an examination of assumptions in the single root model. Soil Sci. 120, 57-67 (1975)

Truesdell, C., Toupin, R.A.: The classical field theories. In: Encyclopedia of Physics, Vol. III/1, pp. 226-794 (1960)

Van Genuchten, M.Th.: A numerical model for water and solute movement in and below the root zone. Research Report no. 121, USDA-ARS, US Salinity Laboratory, Riverside CA, USA (1987)

Van Genuchten, M.Th., Leij, F.J., Lund, L.J. (eds.): Indirect methods for Estimating the Hydraulic Properties of Unsaturated Soils, Proceedings of an International Workshop, organized by the U.S. Salinity Laboratory, USDA-ARS, and the Department of Soil and Environmental Sciences of the University of California, both at Riverside, CA, USA, and held 11-13 Oct. 1989 at Riverside, CA, USA, Univ. of California, Riverside, CA, USA (1992)

Van Genuchten, M.Th., Leij, F.J., Wu, L. (eds.): Characterization and Measurement of the Hydraulic Properties of Unsaturated Porous Media. Proceedings of an International Workshop organized by the U.S. Salinity Laboratory, USDA-ARS, and the Department of Environmental Sciences of the 
University of California, both at Riverside, CA, USA, and held 22-24 October, 1997 at Riverside, CA, USA, Univ. of California, Riverside, CA, USA, 2 Vols. (1999)

Van Noordwijk, M.: Functional interpretation of root densities in the field for nutrient and water uptake. In: Wurzelökologie und ihre Nutzanwendung - Root Ecology and its Practical Application, Int. Symp. Gumpenstein, 1982, Bundesanstalt Gumpenstein, Irdning, Bundesanstalt für alpenländische Landwirtschaft, pp. 207-226, Irdning, Austria (1983)

Van Noordwijk, M.: Methods for quantification of root distribution pattern and root dynamics in the field. Methodology in Soil-K Research, Proc. 20th Colloq. Intern. Potash Inst., Bern, pp. 263-281 (1987)

Van Noordwijk, M., Brouwer, G.: Review of quantitative root length data in agriculture. In: McMichael B.L., Persson H. (eds.) Developments in Agricultural and Managed-Forest Ecology no 24, Plant Roots and their Environment, pp. 515-525. Elsevier, Amsterdam (1991)

Veen, B.W., Van Noordwijk, M., De Willigen, P., Boone, F.R., Kooistra, M.J.: Root-soil contact of maize, as measured by a thin-section technique, III Effects on shoot growth, nitrate and water uptake efficiency. Plant Soil 139, 131-138 (1992)

Whitaker, S.: Flow in porous media II: the governing equations for immiscible, two-phase flow. Transport Porous Media 1, 105-125 (1986)

Zeng, X.: Global vegetation root distribution for land modeling. J. Hydrometeorol. 2, 525-530 (2001)

Zeng, X., Dai, Y.-J., Dickinson, R.E., Shaikh, M.: The role of root distribution for climate simulation over land. Geophys. Res. Lett. 25, 4533-4536 (1998) 